\title{
Design and implementation of wireless sensor networks for impedance-based structural health monitoring using ZigBee and Global System for Mobile Communications
}

Journal of Intelligent Material Systems and Structures

20I5, Vol. 26(I0) 1207-1218

(C) The Author(s) 2014

Reprints and permissions:

sagepub.co.uk/journalsPermissions.nav DOI: I0.II77/I045389X|4538532 jim.sagepub.com (SSAGE

\author{
Nicolás E Cortez ${ }^{1,2}$, Jozué Vieira Filho ${ }^{2,3}$ and Fabricio G Baptista ${ }^{4}$
}

\begin{abstract}
This article presents the design and implementation of a novel wireless structural health monitoring system based on the electromechanical impedance principle. The proposed system is a wireless low-power scalable sensor network composed of multiple sensor nodes and a link node used as master to establish communication with the remote monitoring center (host node and server). The link node communicates with the remote monitoring center through a Global System for Mobile Communications/General Packet Radio Services network and with other sensor nodes through a ZigBee network. Each sensor node is a portable and autonomous structural health monitoring core based on microcontroller, digital synthesizer, and transceiver ZigBee. The identification of damage is performed by simply comparing the variations in root mean square voltage obtained from piezoelectric transducers, such as lead zirconate titanate patches, bonded to the structure. The lead zirconate titanate patches are excited on a wideband frequency range, and it is not necessary to compute the electromechanical impedance. The proposed system was built and experiments were carried out on an aluminum structure, and excellent results have been obtained. Our contribution ranges from the hardware to the graphical front end.
\end{abstract}

\section{Keywords}

Structural health monitoring, impedance, wireless, piezoelectric sensor

\section{Introduction}

A wide variety of structures has still been used despite the long lifetime and damage possibilities, which could affect their performance or harm the safety of users. Therefore, structural health monitoring (SHM) systems using appropriate damage detection techniques have concerned a large number of academic and industrial researchers and become nowadays a very important field of research from both economic and safety perspectives (Ostachowicz and Güemes, 2013).

The main purpose of SHM systems is to monitor a structure and detect incipient damage in normal operation condition using nondestructive evaluation (NDE), nondestructive testing (NDT), or nondestructive inspection (NDI), which can improve the regular operation and maintenance of different types of structures (Giurgiutiu, 2013). In this context, SHM method based on the electromechanical impedance (EMI) technique has been regarded as one of the most promising methods, and it is distinguished by its simplicity and by using low-cost components, such as piezoelectric transducers of lead zirconate titanate (PZT) (Annamdas and Radhika, 2013; Park et al., 2003).

The basic principle of the EMI technique is based on the piezoelectric effect, which is the property that some special materials have to convert mechanical energy into electrical energy (direct effect) and electrical

\footnotetext{
'Departamento Académico de Matemática y Física, Universidad Nacional de San Cristóbal de Huamanga, Ayacucho, Perú

${ }^{2}$ Faculdade de Engenharia, UNESP - Univ Estadual Paulista, Câmpus de Ilha Solteira, Departamento de Engenharia Elétrica, Ilha Solteira, Brazil

${ }^{3}$ Câmpus Experimental de São João da Boa Vista, UNESP - Univ Estadual Paulista, Engenharia de Telecomunicações, São João de Boa Vista, Brazil ${ }^{4}$ Faculdade de Engenharia, UNESP - Univ Estadual Paulista, Câmpus de Bauru, Departamento de Engenharia Elétrica, Bauru, Brazil
}

\section{Corresponding author:}

Fabricio G Baptista, Faculdade de Engenharia, UNESP, Câmpus de Bauru, Departamento de Engenharia Elétrica, Laboratœrio de Transdutores e Aquisição de Dados, Bauru - SP, I7033-360, Brazil.

Email: fabriciogb@feb.unesp.br 
energy into mechanical energy (reverse effect). When a piezoelectric transducer is bonded to the structure to be monitored, there is an interaction between the mechanical impedance of the host structure and the electrical impedance of the transducer. Therefore, changes in the mechanical impedance of the host structure caused by damage, such as cracks or corrosion, can be evaluated simply by measuring the electrical impedance of the transducer in a suitable frequency range (Baptista and Vieira Filho, 2010; Peairs et al., 2007) and using appropriate excitation signal (Baptista et al., 2010). The electrical impedance signatures must be acquired at a highfrequency range in order to limit the actuation/sensing area of the transducer and make the impedance signatures more sensitive to minor changes or incipient damage of the monitored structure (Chaudhry et al., 1995).

Typical SHM applications based on EMI generally use expensive commercial impedance analyzers or alternative systems based on frequency response function (FRF) or other methods. These systems are often prohibitive for many applications because they require data acquisition (DAQ) devices with high sampling rate, digital signal processors (DSP), large data storage capabilities, and personal computer (PC) to process information or they are limited by the sampling rate of the analog-to-digital converters (ADCs) (Neto et al., 2011; Panigrahi et al., 2010; Radil et al., 2008; Wang and You, 2008).

As promising alternative, a new microcontrolled SHM system based on the EMI principle was recently proposed, where the monitoring is only performed by detecting variations in the root mean square (RMS) voltage of PZT transducers bonded to the structure, not being necessary to store the data of the excitation signal. The PZT is excited by sinusoidal signals in an appropriate frequency range, and the system is not limited by the sample rate of the ADC (Cortez et al., 2013).

On the other hand, as the size of the monitoring system grows (increase in the number of sensors or actuators and their distribution in a structure), additional cabling may result in significant increases in installation time and expense. As a result, such monitoring systems tend to present a low spatial density of sensors across the networks. This has motivated the structural monitoring community to seek alternative methods to traditional wired systems that would allow for a dense deployment of sensors with greater affordability (Peckens et al., 2014). In comparison with their natural counterparts (i.e. wired monitoring systems), the wireless sensor networks (WSNs) hold the promise of revolutionizing sensing in a wide range of application domains because of their reliability, time synchronization, accuracy, flexibility, cost-effectiveness, local data processing, and ease of deployment. Thus, WSNs have been widely explored for use in SHM applications
(Araujo et al., 2012; Farrar et al., 2009; Lynch and Loh, 2006; Sazonov et al., 2010; Tanner et al., 2003).

Practical implementations of sensor networks in monitoring systems were performed by different researchers. For the monitoring of excavation for the transit station construction in Singapore, for example, very long input/output wires were used to connect the sensors with the monitoring instrument (electrical impedance analyzer), up to $400 \mathrm{~m}$ (Annamdas and Yang, 2012). The authors have shown that the electrical admittance signatures are influenced by the length of input/output wires, and consequently, the position of major peaks and their magnitudes change with the length of wire and can reduce the accuracy of the results. Numerous studies have effectively overcome such challenges in a variety of infrastructure systems, such as bridges which have had numerous successful deployments of WSNs (Peckens et al., 2014). Taylor et al. (2010) presented a development related to compact hardware and wireless impedance sensor node for use in high-frequency EMI-based SHM (up to $100 \mathrm{kHz}$ ) and low-frequency vibration DAQ.

However, some drawbacks such as instability, power consumption, and insufficient bandwidth are the key problems when designing WSNs. To overcome these problems, WSNs based on ZigBee (IEEE 802.15.4) technology are available. Compared to some conventional wireless personal area networks (WPANs), such as Bluetooth (IEEE 802.15.1) or radio frequency identification (RFID), a ZigBee radio interface presents some advantages, such as low power consumption, long data rate, and reliable and different application topologies (Araujo et al., 2012; Krishnamurthy and Sazonov, 2008; ZibBee Alliance, 2008). Other WSN characteristics based on ZigBee technology applied to SHM are discussed in Chen et al. (2012), Harms et al. (2009), Nanda et al. (2012), and Wang et al. (2012).

This study aims to contribute to the development and implementation of an impedance-based SHM system using WSN. The proposed system is wireless and low-power sensor network, composed of a link node to establish communication between remote monitoring center (host node and server) and multiple sensors nodes. The link node communicates with the remote monitoring center through a Global System for Mobile Communications (GSM)/General Packet Radio Services (GPRS) network and with the sensor node through a ZigBee network. A GSM modem provides long-range communication capability using GSM cellular network and allows the SHM system to be used virtually from anywhere in the world. Each sensor node is a portable and autonomous SHM core based on microcontroller, digital synthesizer, and wireless transceiver ZigBee. Damage detection is performed by comparing RMS variations computed from response signals of piezoelectric transducers, such as PZT patches bonded 


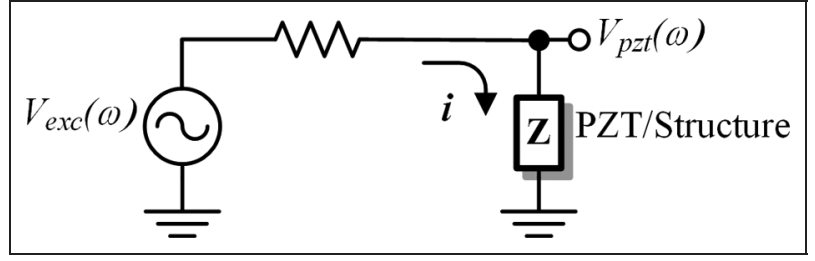

Figure I. Excitation circuit to PZT/structure.

PZT: lead zirconate titanate.

to the structure, without computing the EMI (Cortez et al., 2013).

After this "Introduction" section, this article is organized as follows. In the next section, we present a brief review of the principle of detecting structural damage based on the variation in the RMS voltage of PZT transducers. Then, the design and implementation of the proposed system are discussed. Finally, we present the analysis of the experimental results and the conclusions.

\section{Damage detection based on RMS voltage variation of piezoelectric transducers}

For a PZT transducer bonded to a structure under an excitation frequency $\left(f_{\text {exc }}\right)$, a direct and simple way to calculate the magnitude of impedance $(|Z(\omega)|)$ is by using the following equation (Nilsson and Riedel, 2011)

$$
|Z(\omega)|=\frac{V_{p z t \_r m s}(\omega)}{I_{p z t \_r m s}(\omega)}
$$

where $V_{p z t \_r m s}(\omega)$ is the RMS excitation voltage, $I_{p z t \_r m s}(\omega)$ is the RMS current, and $\omega=2 \pi f_{\text {exc }}$ is the angular frequency.

Equation (1) shows that the magnitude of the impedance can be obtained simply by measuring the RMS voltage and the corresponding RMS current, which is the principle used in this study. From this basic concept, we can use the simple circuit shown in Figure 1 to relate the EMI and the RMS voltage of the response signal from the PZT transducer.

Since the PZT transducer is a predominantly capacitive device and considering that the excitation signal is a pure sinusoidal wave at a frequency high enough, the phase difference between voltage and current tends to be small, and the magnitude of the impedance is approximately given by

$$
|Z(\omega)| \cong \frac{V_{p z t \_r m s}(\omega)}{V_{\text {exc_rms }}(\omega)-V_{p z t \_r m s}(\omega)} R_{S}
$$

where $R_{S}$ is a current limiter resistor, $|Z(\omega)|$ is the magnitude of the EMI, and $V_{\text {exc_rms }}(\omega)$ and $V_{p z t \_r m s}(\omega)$ are the RMS voltage of the excitation signal and response signal, respectively.
Solving equation (2) for the RMS voltage of the transducer, we obtain

$$
V_{p z t \_r m s}(\omega) \cong \frac{|Z(\omega)|}{|Z(\omega)|+R_{S}} V_{\text {exc_rms }}(\omega)
$$

From equation (3), considering steady state and a constant excitation signal for each frequency $(\omega)$, we conclude that the RMS voltage $\left(V_{p z t \_r m s}(\omega)\right)$ of the transducer changes only if the magnitude of EMI $(Z(\omega))$, which is directly related to the physical condition of the monitored structure, also changes.

Therefore, the RMS voltage is sensitive to any change in the mechanical impedance of the structure, and this approach is sufficient to detect structural damage. This methodology has been confirmed experimentally, and the results indicate that it is possible to develop a simple SHM system based on EMI, only monitoring the RMS voltage of the PZT transducer in an appropriate frequency range. This result was recently analyzed and discussed by Cortez et al. (2013).

The approach implemented in this proposed system performs DAQ independent of the sampling rate. However, in order to verify the success of this approach, the proposed system was implemented to detect damage in the frequency range between 0 and $250 \mathrm{kHz}$. Moreover, being provided of a 12-bit ADC with reference voltage of $2.5 \mathrm{~V}$, it is possible to detect changes of $0.6 \mathrm{mV}$ in the RMS voltage signatures, allowing to monitor damage with great accuracy.

Figure 2 shows the normalized RMS voltage signatures obtained using the proposed system and normalized impedance obtained using a reference system developed by Baptista and Vieira Filho (2009) in a frequency range of (1) $17-33 \mathrm{kHz}$ and (2) $120-200 \mathrm{kHz}$. These measurements were performed using a simple rectangular aluminum beam of $500 \times 30 \times 2 \mathrm{~mm}^{3}$ as the test specimen (monitored structure). The frequency range of $17-33 \mathrm{kHz}$ was determined experimentally as the most sensitive to damage. However, the RMS voltage signature shows a good match with the impedance signature for higher frequencies, as shown in Figure 2(b), and it is also sensitive to damage.

The system performs the calculation of damage indices comparing the updated RMS voltage signature with the baseline signature using two indices: the root mean square deviation (RMSD) and the correlation coefficient deviation metric (CCDM). The RMSD index is based on Euclidean norm and is given by (Giurgiutiu and Rogers, 1998)

$$
R M S D=\sum_{n}^{N} \sqrt{\frac{\left(V_{n, d}-V_{n, h}\right)^{2}}{V_{n, h}{ }^{2}}}
$$

where $V_{n, h}$ and $V_{n, d}$ are the RMS voltage signatures for the structure in healthy (baseline) and damaged 


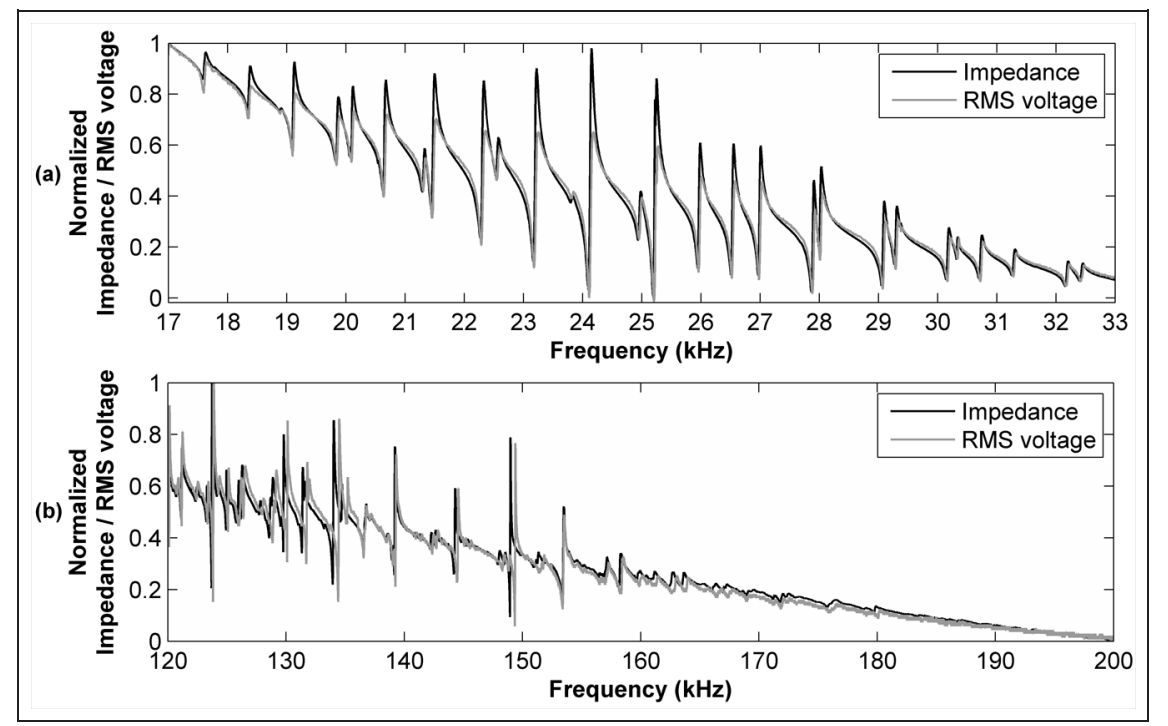

Figure 2. Qualitative comparison between the RMS voltage and the impedance of the PZT transducer for (a) low frequencies and (b) high frequencies. Both signatures were normalized between the 0 and I limits. RMS: root mean square; PZT: lead zirconate titanate.

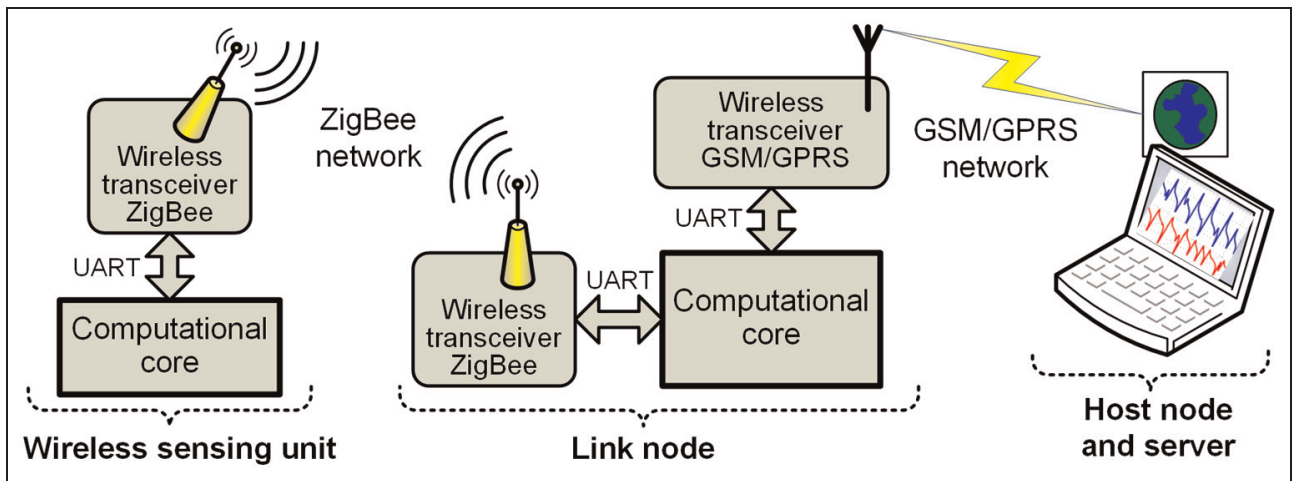

Figure 3. Functional diagram of the proposed impedance-based wireless SHM system. SHM: structural health monitoring; UART: Universal Asynchronous Receiver and Transmitter; GSM: Global System for Mobile Communications; GPRS: General Packet Radio Services.

conditions, respectively, both measured at frequency $n$, and $N$ is the total number of samples.

The CCDM index is given by (Marqui et al., 2008)

$$
C C D M=1-C_{C}
$$

where $C_{C}$ is the correlation coefficient calculated using the RMS voltage signatures, which can range.

\section{Proposed wireless SHM system}

The prototype of the impedance-based SHM system incorporates an integrated hardware and software design to implement a WSN. As shown in Figure 3, the proposed system is composed of three functional nodes: (1) wireless sensing unit (WSU), (2) link node, and (3) remote monitoring center (host node and server).

The proposed system is controlled by the remote monitoring center (laptop or desktop), which communicates with the link node through a GSM/GPRS network. The link node coordinates communication between remote monitoring center and multiple WSUs.

The proposed wireless SHM system implements a simple star-topology ZigBee network using multiple WSUs connected to the link node, where the link node is the network coordinator. ZigBee's addressing scheme 


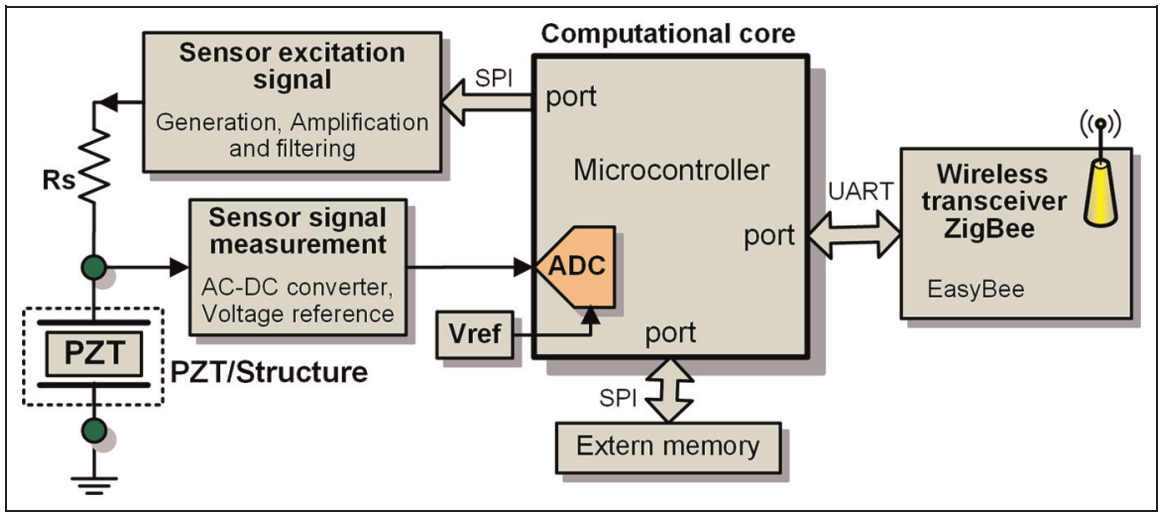

Figure 4. Functional diagram of the wireless sensing unit (WSU). PZT: lead zirconate titanate; AC: alternating current; DC: direct current; SPI: Serial Peripheral Interface; UART: Universal Asynchronous Receiver and Transmitter; ADC: analog-to-digital converter.

is capable of supporting more than 64,000 WSUs per network. The WSUs are responsible for acquiring sensor measurements and analyzing data and through link node perform data transfer to central server for permanent storage or further data interrogation.

\section{Hardware of a WSU}

Each WSU represents an autonomous node within the wireless SHM system, collecting and analyzing measurements from PZT transducer. A functional diagram of the proposed WSU is illustrated in Figure 4.

The design of the WSU consists of four functional modules: (1) sensor excitation signal, (2) sensor signal measurement, (3) computational core, and (4) wireless transceiver ZigBee. The sensor excitation signal module provides a sinusoidal wave synthesized by a Digital Direct Synthesizer (DDS) device and amplified and filtered before being applied to the PZT transducer.

The sensor signal measurement module performs the alternating current (AC) to direct current (DC) conversion of the response signal from the PZT transducer. The computational core is responsible for configuring and controlling the frequency sweep of the signal generated by the DDS and for each frequency point converts analog RMS voltage from transducer into a digital format. The RMS voltage digitized is then transferred to external memory to accommodate local data storage, analysis, and subsequent retransmission to remote monitoring center. Through a Universal Asynchronous Receiver and Transmitter (UART) interface, the computational core communicates with the wireless transceiver ZigBee, which provides a wireless connection between the WSU and the link node.

The sensor excitation signal module is composed of a signal generator based on DDS and a Butterworth low-pass filter (LPF) with voltage gain as signal conditioner. The sensor signal measurement module is basically a precision rectifier circuit required to perform the
AC-DC conversion of the response signal from the transducer, providing at its output the RMS voltage corresponding to each excitation frequency generated by the DDS device.

Details of these hardware designs have been described by Cortez et al. (2013). This section describes only the hardware design of computational core and wireless transceiver ZigBee module. Figure 5(a) shows the prototype of the WSU, the aluminum specimen, and the steel nut (load) used in the tests.

Computational core of the WSU. For the computational core of the WSU, a microcontroller is employed to coordinate all different parts of the sensing unit hardware and to provide the capability for local data interrogation. A low-cost 8-bit microchip microcontroller PIC18F2553 with power consumption of about $70 \mathrm{~mW}$ in active mode and only $0.5 \mu \mathrm{W}$ in sleep mode was chosen in this design. The PIC18F2553 microcontroller provides $32 \mathrm{kB}$ of in-system reprogrammable flash memory, which is sufficient for storing embedded programs for some typical computational algorithms, such as to compute the RMSD and CCDM indices. The 2 $\mathrm{kB}$ static random-access memory (SRAM) integrated in the microcontroller is insufficient for sensor data storage and analysis; therefore, the microcontroller is interfaced with an external $128 \mathrm{kB}$ memory microchip 25LC1024. This external memory is sufficient for storing a large quantity of sensor data, such as the data corresponding to a sweep in a frequency range of $5-250 \mathrm{kHz}$ with increments of $4 \mathrm{~Hz}$.

To coordinate the behavior of the WSU, embedded system software is written for the PIC18F2553 microcontroller. Running the embedded system software, the microcontroller performs the following tasks: (1) receives DDS configuration parameters from the link node through wireless transceiver ZigBee, (2) configures DDS parameters and controls the frequency sweep 


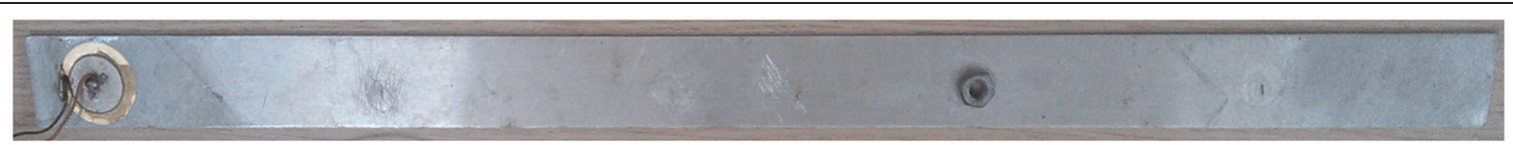

(a)

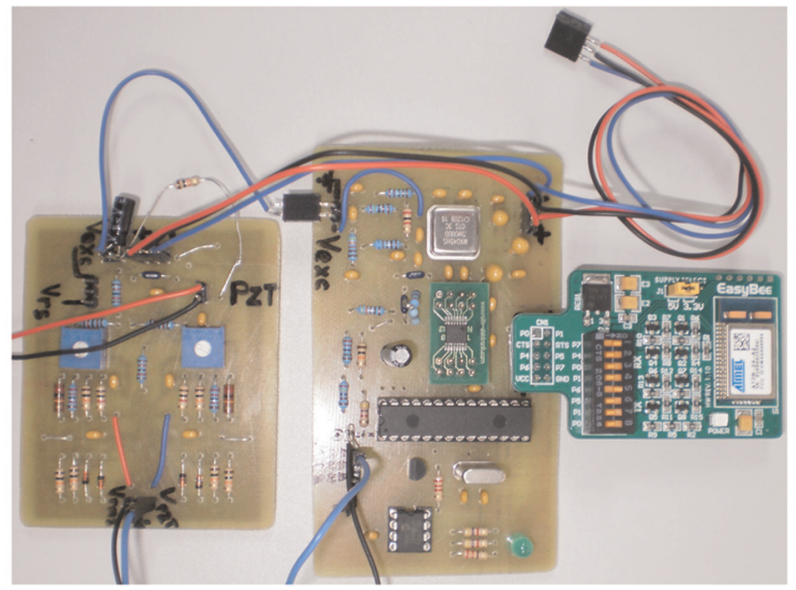

(b)

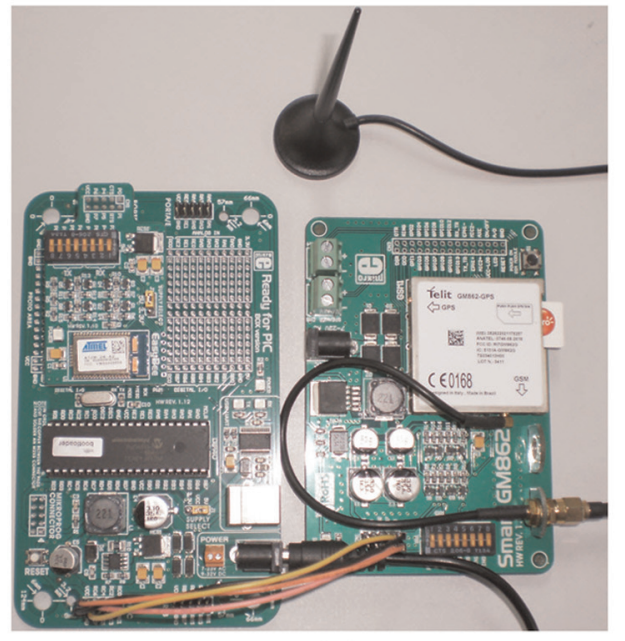

(c)

Figure 5. (a) Structure/PZT and steel nut, (b) wireless sensing unit (WSU) prototype, and (c) link node prototype. PZT: lead zirconate titanate.

of the excitation signal generated by DDS via Serial Peripheral Interface (SPI) communication interface, (3) collects data corresponding to the PZT RMS voltage from the sensor signal measurement module using an embedded 12-bit ADC, and (4) stores data into external memory via SPI communication interface. When the frequency sweep is completed, the microcontroller executes computational algorithm to interrogate sensor data, evaluating the CCDM and RMSD indices, and subsequently transmits the data to the link node via wireless transceiver ZigBee module.

It is important to note that the ADC sampling rate of the computational core is only $50 \mathrm{kS} / \mathrm{s}$. However, the acquisition of data is not limited by the sampling rate since the signatures are based on the RMS voltage (a DC value), as described previously.

Wireless transceiver ZigBee module. In some applications, the communication range can be upwards of several hundred meters. However, long communication ranges usually require higher power consumption of the wireless transceiver. In this study, the communication wireless unit is the MikroElektronika EasyBee module, which was chosen because it offers appropriate tradeoff and balance between low power consumption (about $320 \mathrm{~mW}$ in TX (transmitter) or RX (receiver) mode and $30 \mu \mathrm{W}$ in sleep mode) and short communication distance for applications in SHM. The EasyBee module comprises mainly an Atmel ZigBit ATZB-24-A2 transceiver, compatible with IEEE 802.15.4/ZigBee and can be utilized worldwide operating in the unlicensed 2.4 $\mathrm{GHz}$ industrial, scientific, and medical (ISM) frequency band (Atmel Corporation, 2008). The computational core controls EasyBee module using AT commands via UART interface set to $19.2 \mathrm{kbps}$ (MeshNetics, 2008).

This module is typically capable of wireless communication over a range of $100 \mathrm{~m}$, using a $2.4 \mathrm{GHz}$ 802.15.4 transceiver (Harms et al., 2009). According to the specifications, the ZigBit transceiver drains about $18 \mathrm{~mA}$ when transmitting data or about $19 \mathrm{~mA}$ when receiving data. In this work, a data frame is organized as 92 decimal digits corresponding to the RMS voltage of 23 frequency points (where the RMS voltage is represented with 4 decimal digits), except in the last frame where only the remaining data are sent.

The baud rate used to send data from the sensor module to the link module is $19.2 \mathrm{kbps}$, which provided consistent and reliable results. Using this data transfer rate, the block of data corresponding to a sweep of 4096 frequency points is transmitted in an estimated time of $8 \mathrm{~s}$.

\section{Hardware of the link node}

The link node is used to integrate both ZigBee network and GSM/GPRS network in order to improve the short-range communication of the WSUs, allowing support for remote control via Internet from anywhere in the world. The link node also represents an 
autonomous node within the wireless SHM system, coordinating communication between remote monitoring center and multiple WSUs. A functional diagram of the proposed link node is illustrated in Figure 3, and Figure 5(b) shows the prototype of the link node.

The design of the link node consists of three functional modules: (1) wireless transceiver ZigBee, (2) computational core, and (3) wireless transceiver GSM/ GPRS. Through a UART interface, the computational core communicates with the wireless transceiver ZigBee, which provides a wireless connection between the link node and the WSUs. Using another UART interface, the computational core communicates with the wireless transceiver GSM/GPRS, which provides a wireless connection between the unit and the remote monitoring center.

Computational core of the link node. A low-cost 8-bit microchip microcontroller PIC18F45K22 was chosen as computational core in this design. The PIC18F45 K22 microcontroller provides $32 \mathrm{kB}$ of in-system reprogrammable flash memory, 1536-byte SRAM, and two UART interfaces.

To coordinate the behavior of the link node, embedded system software is written for the PIC18F45 K22 microcontroller. Executing the embedded system software, the microcontroller performs the following tasks: (1) retransmits control parameters to the WSUs from remote monitoring center and (2) retransmits SHM data to the server central from the WSUs.

Wireless transceiver GSM/GPRS module. The communication wireless unit is the MikroElektronika SmartGM 862 module. This module is mainly composed of a Telit GM862-GPS quad-band transceiver with support to operate in the unlicensed $(850,900,1800$, and 1900 $\mathrm{MHz}$ ) ISM frequency bands (Telit Communications, 2011). The SmartGM 862 module provides long-range communication capability using GSM cellular network. The computational core controls SmartGM862 module using AT commands via UART interface set to 38.4 kbps (Telit Communications, 2012).

\section{Software}

As previously mentioned, the system includes one remote monitoring center, a link node, and multiple WSUs. The central server is responsible for commanding WSUs to perform DAQ tasks and storing the measurement data in a file server for subsequent analysis. The link node coordinates the communication between central server and WSUs.

The software written for the wireless SHM system is divided into three parts: (1) computer software for the remote monitoring center, (2) embedded software for the link node, and (3) embedded software for the
WSUs. Since the remote monitoring center and the WSUs must frequently communicate with each other, portions of their software are designed to allow seamless integration and coordination. A state diagram is constructed to describe in detail the program flow for both the link node and the WSU. The aim of the state diagram is to encode a sequence of actions for both the link node server and the WSUs so that all potential problematic scenarios (or states) commonly encountered in an unreliable wireless channel can be efficiently handled.

\section{Computer software for the remote monitoring center}

The communication is always initiated by the remote monitoring center. Figure 6 shows the user-friendly interface of the remote monitoring center. Basically, the user must first set the values of the frequency sweep in the text boxes "Start Frequency," "Step Frequency," and "End Frequency" and set the sensor number. The swap area used for temporary storage of control commands, configuration parameters, and data storage are tables implemented into a database installed on the server.

A display allows the comparison of the baseline and updated RMS voltage signatures, and the interface provides the corresponding RMSD and CCDM indices. This interface was developed to analyze a specific experiment, where structural damage was induced in an aluminum bar by bonded a small steel nut at distances of $10,20,30$, and $40 \mathrm{~cm}$ from the PZT patch. However, the interface can be easily modified for a general purpose application.

\section{Embedded software for the link and sensor nodes}

For many damage and system identification procedures, measurement data individually collected by different sensors must be time synchronized. The design of the communication protocol between the link node and the WSUs uses the state machine concept. A state machine consists of a set of states and the definition of a set of transitions among these states. At any instant of time, the state machine can be in only one of the possible states. In response to different events, the state machine transits between its discrete states. Figure 7(a) shows the simplified state diagram for the WSUs software, and Figure 7(b) shows the simplified state diagram for the link node software.

In the state diagrams shown in Figure 7, each circle with bold boundary line stands for one possible state; lines with arrows represent state transitions. As shown in the legends, for each transition, the normal text above the horizontal line specifies the event/condition upon which the transition should happen, and the italic text below the horizontal line specifies the service/ action to be completed during this transition. 


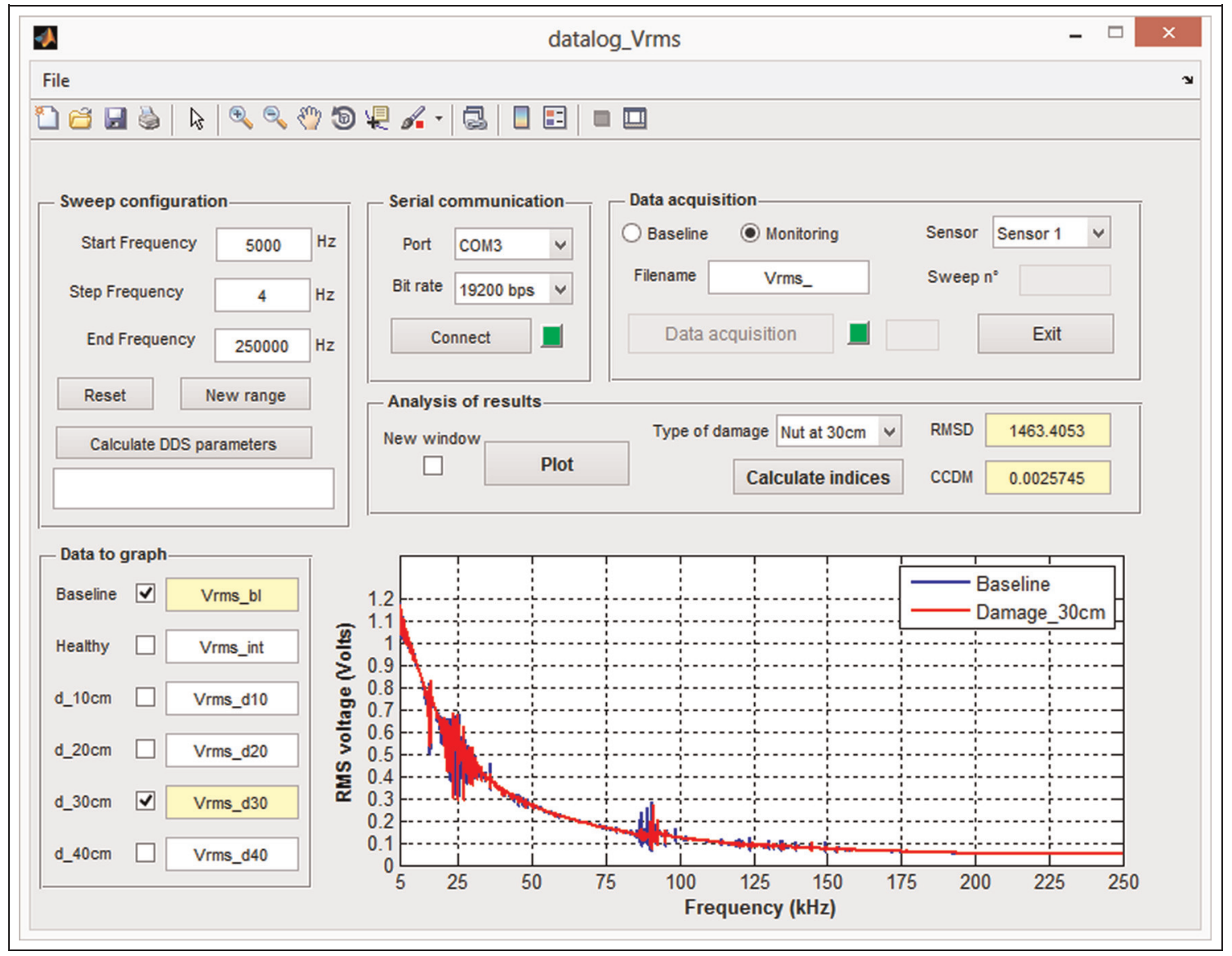

Figure 6. User-friendly interface of the remote monitoring center.

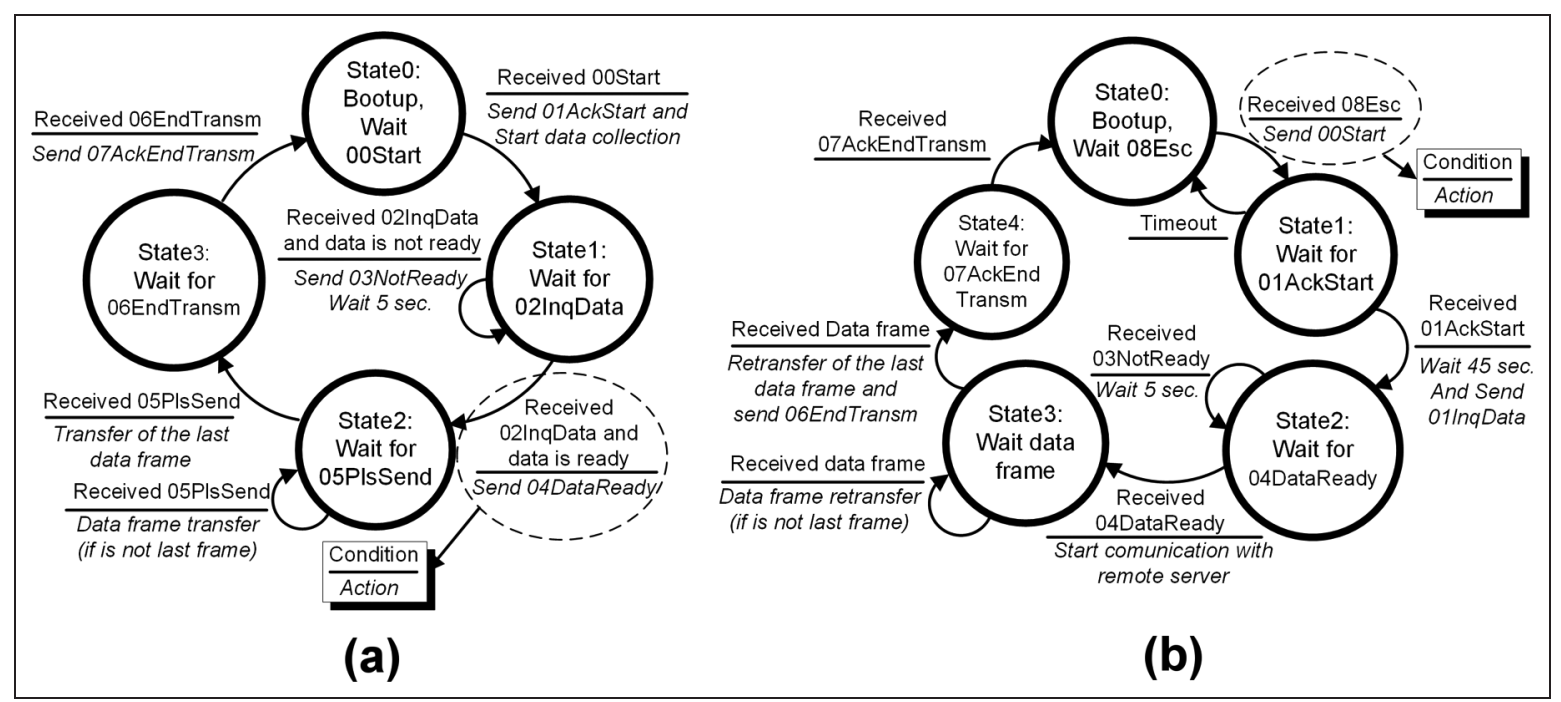

Figure 7. Simplified communication state diagrams for (a) the WSU and (b) the link node.

When a WSU is powered on, the unit starts from "State0: Bootup and Wait for 00Start" in Figure 7(a). "00Start" is the beacon signal that is broadcasted from the link node requesting one between various units to start DAQ. As soon as the WSU receives and recognizes the "00Start" beacon, it sends "01AckStart" to link node, starts collecting data, saves the data temporarily into its external memory for later acquisition by 
the link node, and transits into "Statel: Wait for 02InqData." Being in the "Statel," if the WSU receives "02InqData" and data collection is not ready, it sends "03NotReady" to link node and restart its "Statel." If data collection is ready, the WSU sends "03DataReady" to link node and immediately transits into "State2: Wait for 05PlsSend." Being in the "State2," the WSU transmits data to link node from external memory. Whenever the WSU receives "05PlsSend," it sends the data frame to link node. When the last data frame is transmitted, the WSU changes its state to "State3: Wait for 06EndTransm." Being in the "State3," if the WSU receives "06EndTransm," it sends "07AckEndTransm" to link node and return at its "State0."

When the link node is powered on, it starts from "State0: Bootup and Wait for 08Esc" in Figure 7(b). "08Esc" is the beacon signal requesting one between various WSUs to start DAQ. As soon as the link node receives and recognizes the "08Esc" beacon, it sends "00Start" to the corresponding WSU and transits into "State1: Wait for 01Ackstart." Being in the "State1" and within the interval of $5 \mathrm{~s}$, the link node receives "01AckStart" from the WSU. Then, the link node waits for $45 \mathrm{~s}$ before sending "01InqData" to the WSU and transits into "State2: Wait for 04DataReady." If timeout occurs, the link node returns at its "State0." Being in the "State2" and if the link node receives "04DataReady" from the WSU, it starts communication with remote server and transits into "State3: Wait for data frame." Otherwise, if the link node receives "03NotReady," it waits $5 \mathrm{~s}$ and restarts its "State2." Being in the "State3," the link node retransmits to the central server all frames received from the WSU. When the last data frame is retransmitted to the central server, the link node sends "06EndTransm" to the WSU and transits into "State4: Wait for 07AckEndTransm." Finally, being in the "State4" and if the link node receives "07AckEndTransm," it returns at its "State0."

\section{Experimental setup}

In order to evaluate the performance of the proposed wireless system, tests were carried out on aluminum beam specimen (monitored structure) of $500 \times 30 \times$ $2 \mathrm{~mm}^{3}$ of $86.72 \mathrm{~g}$, as shown in Figure 5(a). A circular PZT transducer with diameter of $19.7 \mathrm{~mm}$ and thickness of $0.24 \mathrm{~mm}$ was bonded to one end of the beam using cyanoacrylate glue. Structural damage was induced in the structure by placing a load (small steel nut of $3.16 \mathrm{~g}$ ) at different distances from the PZT transducer. The load represents $3.64 \%$ of the weight of the aluminum plate. This method is commonly used in the literature and has the advantage of not causing permanent damage on the specimen.

In this study, we used only one WSU, which was installed with the monitored structure at a distance of
$35 \mathrm{~m}$ from the link node using the internal antenna design. The processing of the RMS voltage signatures and calculation of damage indices were performed on a PC notebook via GSM using the interface shown in Figure 6 .

\section{Results}

First, the RMS voltage signature was obtained for the structure in healthy condition (without load), which is known as the reference or baseline signature. Then, new RMS voltage signatures were obtained for the structure in damaged condition by placing the load at distances of $10,20,30$, and $40 \mathrm{~cm}$ from the PZT transducer. The RMSD and CCDM indices were computed using equations (4) and (5) for both frequency ranges of $17-33 \mathrm{kHz}$ and $120-140 \mathrm{kHz}$ with steps of $4 \mathrm{~Hz}$. These frequency ranges were determined experimentally as that offered improved sensitivity for detection of damage. However, different ranges can be obtained for other types of structures, transducers, or damage. Furthermore, the proposed system allows analyzing the structure at frequencies from DC up to $250 \mathrm{kHz}$. Higher frequencies can be obtained by an appropriate design.

Figure 8 shows the RMS voltage signatures obtained for the structure in the healthy and damaged conditions for the two frequency ranges mentioned above. Only narrow bands of (1) $23-24 \mathrm{kHz}$ and (2) $130-131 \mathrm{kHz}$ are displayed to allow a reasonable comparison between the signatures.

According to Figure 8, we observe that the presence of load caused substantial variation in RMS voltage signatures, particularly for load localized at distances of 30 and $10 \mathrm{~cm}$ from the PZT transducer for the lowand high-frequency ranges, respectively. These results confirm that the RMS voltage is sensible and feasible to detect structural damage and that data were successfully sent to the host node.

A more accurate comparison is possible through damage indices. Figure 9 shows (a) the RMSD and (b) CCDM indices computed using the frequency ranges of 17-33 and 120-140 kHz. Both the indices were normalized considering the value 1.00 for the healthy condition.

The CCDM index provided better results. The values obtained for the structure in damage conditions were about 120 times higher when compared to the healthy condition for the low-frequency range and about 40 times higher for the high-frequency range. Particularly for the load located at distances of 30 and $10 \mathrm{~cm}$ from the PZT transducer, the ratio was 153 and 53 times higher for the low- and high-frequency ranges, respectively. On the other hand, the values obtained with the RMSD index were lower. The ratio between the damaged conditions and healthy condition is between 11.4 


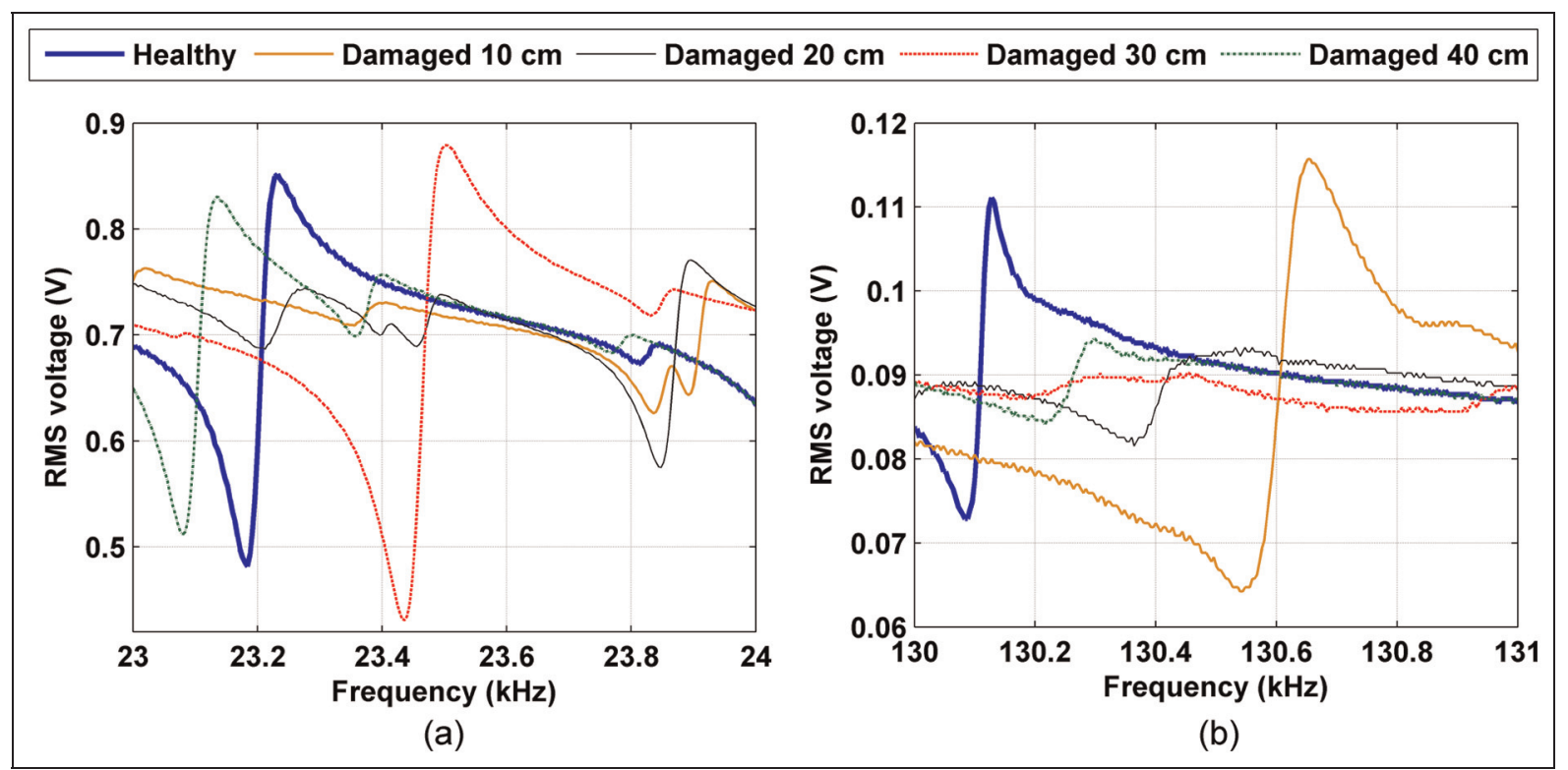

Figure 8. RMS voltage signature obtained for the structure in the healthy and damaged conditions for (a) low-frequency range and (b) high-frequency range.

RMS: root mean square.

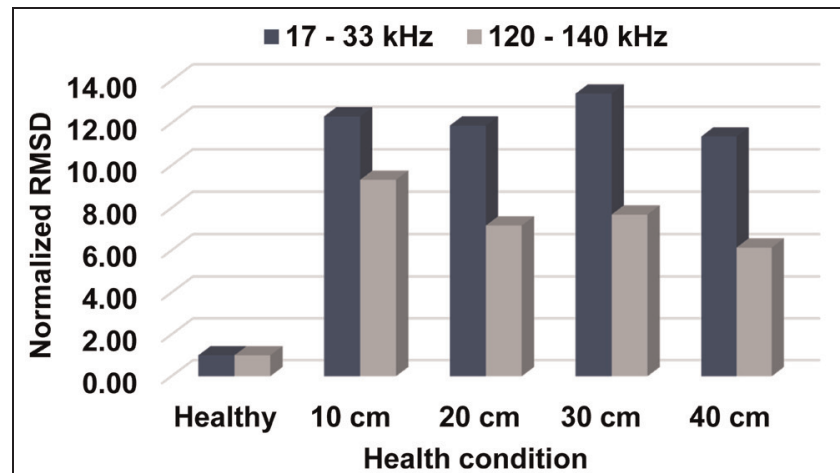

(a)

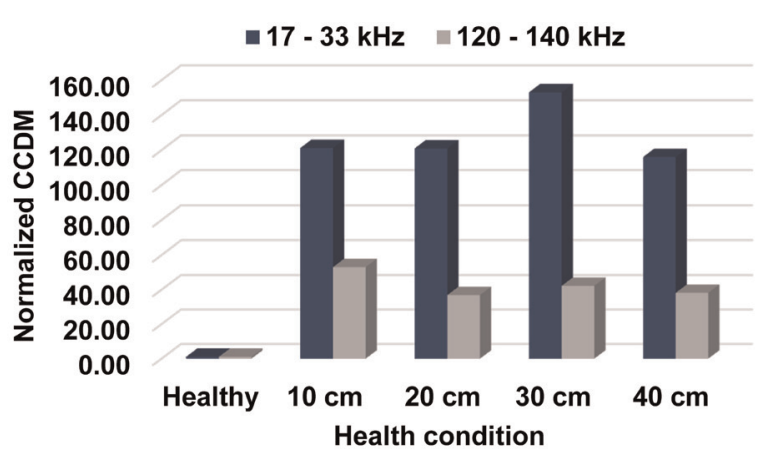

(b)

Figure 9. (a) RMSD and (b) CCDM indices obtained for the structure in the healthy condition and damaged conditions with damage located at distances of $10,20,30$, and $40 \mathrm{~cm}$ from the PZT transducer.

RMSD: root mean square deviation; CCDM: correlation coefficient deviation metric; PZT: lead zirconate titanate.

and 13.4 for the low-frequency range and between 6.1 and 9.3 for the high-frequency range.

Although the indices are lower for the highfrequency range, this does not indicate a deficiency of the proposed system in high frequencies. According to the study presented by Baptista and Vieira Filho (2010), it is expected that indices decrease as frequency increases. Therefore, the experimental results indicate conclusively that the proposed wireless system is feasible to detect structural damage.

\section{Conclusion}

The main goal of this study was to design and implement an integrated novel WSN for impedance-based
SHM system, whereby communication and damage detection algorithms were properly integrated with the sensors, microcontrollers, and wireless transceivers commercially available.

There are two main contributions to wireless SHM systems, which ranges from the hardware to the graphical front end. The first one is the DAQ approach, which does not depend on ADC sampling rate and brings a method for identification of damage that performs a simple comparison between variations in the RMS voltage obtained from response signals of piezoelectric transducers, allowing the development of systems with simpler hardware and software. The second contribution is the WSUs with low-range coverage and ability to scale to a large number of nodes that can be 
monitored worldwide. Therefore, a dense sensor grid operating on real-world structures becomes possible.

\section{Acknowledgements}

The authors are thankful to INCT-EIE and the anonymous reviewers.

\section{Declaration of conflicting interests}

The authors declared no potential conflicts of interest with respect to the research, authorship, and/or publication of this article.

\section{Funding}

This work was partially supported by CNPq, FAPEMIG, CAPES Foundation, and FAPESP (grant 2011/20354-6).

\section{References}

Annamdas VGM and Radhika MA (2013) Electromechanical impedance of piezoelectric transducers for monitoring metallic and non-metallic structures: a review of wired, wireless and energy-harvesting methods. Journal of Intelligent Material Systems and Structures 24(9): 1021-1042.

Annamdas VGM and Yang Y (2012) Practical implementation of piezo-ceramic sensors in monitoring of excavation for transit station construction in Singapore. Structural Control \& Health Monitoring 19(2): 231-245.

Araujo A, García-Palacios J, Blesa J, et al. (2012) Wireless measurement system for structural health monitoring with high time-synchronization accuracy. IEEE Transactions on Instrumentation and Measurement 61(3): 801-810.

Atmel Corporation (2008) ZigBit ${ }^{T M} 2.4$ GHz Wireless Modules. San Jose, CA: Atmel Corporation, 28 pp.

Baptista FG and Vieira Filho J (2009) A new impedance measurement system for PZT-based structural health monitoring. IEEE Transactions on Instrumentation and Measurement 58(10): 3602-3608.

Baptista FG and Vieira Filho J (2010) Optimal frequency range selection for PZT transducers in impedance-based SHM systems. IEEE Sensors Journal 10(8): 1297-1303.

Baptista FG, Vieira Filho J and Inman DJ (2010) Influence of excitation signal on impedance-based structural health monitoring. Journal of Intelligent Material Systems and Structures 21: 1409-1416.

Chaudhry Z, Joseph T, Sun F, et al. (1995) Local-area health monitoring of aircraft via piezoelectric actuator/sensor patches. In: Proceedings of annual international symposium on smart structures and materials, San Diego, CA, 26 February, vol. 2443, pp. 268-276. Washington, DC: SPIE.

Chen H, Zhu L, Huang X, et al. (2012) Structural health monitoring system of Gantry Crane based on ZigBee technology. In: Proceedings of the 3rd international conference on digital manufacturing and automation (ICDMA), Guilin, China, 31 July-2 August, pp. 801-804. New York: IEEE.

Cortez NE, Vieira Filho J and Baptista FG (2013) A new microcontrolled structural health monitoring system based on the electromechanical impedance principle. Structural Health Monitoring 12(1): 14-22.
Farrar CR, Park G and Farinholt KM (2009) Chapter 71: sensor network paradigms. In: Boller C, Chang F and Fujino Y (eds) Encyclopedia of Structural Health Monitoring. Chichester: John Wiley \& Sons, pp. 1251-1268.

Giurgiutiu V (2013) Embedded NDT with piezoelectric wafer active sensors. In: Güneş $\mathrm{O}$ and Akkaya Y (eds) Nondestructive Testing of Materials and Structures. Berlin: Springer Verlag, pp. 987-992.

Giurgiutiu V and Rogers CA (1998) Recent advancements in the electro-mechanical (E/M) impedance method for structural health monitoring and NDE. In: Proceedings of annual international symposium on smart structures and materials (5th SPIE), San Diego, CA, 27 July, vol. 3329, pp. 536-547. Washington, DC: SPIE.

Harms T, Shah PV, Sedigh S, et al. (2009) ZigBee-enabled structural health monitoring with the Smart Brick network. In: Proceedings of the 7th international workshop on structural health monitoring, Stanford, CA, 9-11 September, pp. 909-916. Lancaster, Pennsylvania, USA: DEStech Publications, Inc.

Krishnamurthy V and Sazonov E (2008) Reservation-based protocol for monitoring applications using IEEE 802.15.4 sensor networks. International Journal of Sensor Networks 4(3): 155-171.

Lynch JP and Loh KJ (2006) A summary review of wireless sensors and sensor networks for structural health monitoring. The Shock and Vibration Digest 38(2): 91-128.

Marqui CR, Bueno DD, Baptista FG, et al. (2008) External disturbance effect in damage detection using electrical impedance. In: Proceedings of international modal analysis conference, Orlando, FL, 4-7 February, no. 26, paper 286. Bethel, Connecticut, USA: SEM - Society for Experimental Mechanics, Inc.

MeshNetics (2008) SerialNet ${ }^{T M}$ Reference Manual: AT-Command Set. Phoenix, AZ: MeshNetics, 71 pp.

Nanda K, Nayak K, Chippalkatti S, et al. (2012) Web based monitoring and control of WSN using WINGZ (wireless IP network gateway for ZigBee). In: Proceedings of the 6th international conference on sensing technology (ICST), Kolkata, India, 18-21 December, pp. 666-671. New York: IEEE.

Neto RMF, Steffen V, Rade DA, et al. (2011) A low-cost electromechanical impedance-based SHM architecture for multiplexed piezoceramic actuators. Structural Health Monitoring 10(4): 391-402.

Nilsson JW and Riedel SA (2011) Electric Circuits. Upper Saddle River, NJ: Prentice Hall, pp. 306-373.

Ostachowicz W and Güemes JA (2013) New Trends in Structural Health Monitoring. Vienna: Springer.

Panigrahi R, Bhalla S and Gupta A (2010) A low-cost variant of electro-mechanical impedance (EMI) technique for structural health monitoring. Experimental Techniques 34(2): 25-29.

Park G, Sohn H, Farrar C, et al. (2003) Overview of piezoelectric impedance-based health monitoring and path forward. The Shock and Vibration Digest 35(6): 451-463.

Peairs DM, Tarazaga PA and Inman DJ (2007) Frequency range selection for impedance-based structural health monitoring. Journal of Vibration and Acoustics: Transactions of the ASME 129(6): 701-719.

Peckens CA, Kane MB, Zhang Y, et al. (2014) Permanent installation of wireless structural monitoring systems in 
infrastructure systems. In: Wang ML, Lynch J and Sohn H (eds) Sensor Technologies for Civil Infrastructures: Sensing Hardware and Data Collection Methods for Performance Assessment. Cambridge: Woodhead Publishing Limited, pp. 5408-5509.

Radil T, Ramos PM and Serra AC (2008) Impedance measurement with sine-fitting algorithms implemented in a DSP portable device. IEEE Transactions on Instrumentation and Measurement 57(1): 197-204.

Sazonov E, Krishnamurthy V and Schilling R (2010) Wireless intelligent sensor and actuator network - a scalable platform for time-synchronous applications of structural health monitoring. Structural Health Monitoring 9(5): 465-476.

Tanner NA, Wait JR, Farrar CR, et al. (2003) Structural health monitoring using modular wireless sensors. Journal of Intelligent Material Systems and Structures 14(1): 43-56.
Taylor SG, Farinholt KF, Park G, et al. (2010) Multi-scale wireless sensor node for health monitoring of civil infrastructure and mechanical systems. Smart Structures and Systems 6(5): 661-673.

Telit Communications (2011) GM862 Family Hardware User Guide. Rev. 2. Sgonico: Telit Communications.

Telit Communications (2012) AT Commands Reference Guide. Rev. 15. Sgonico: Telit Communications.

Wang P, Yan Y, Tian GY, et al. (2012) Investigation of wireless sensor networks for structural health monitoring. Journal of Sensors 2012: 1-7.

Wang S and You C (2008) A circuit design for impedancebased structural health monitoring. Journal of Intelligent Material Systems and Structures 19(9): 1029-1040.

ZibBee Alliance (2008) ZigBee specification. ZigBee Document 053474r17, 17 January. San Ramon, CA: ZibBee Alliance. 